\title{
Optical Coherence Tomography Assessment of Macular and Choroidal Thickness in Patients with Proliferative Diabetic Retinopathy in Relation to Hemoglobin A1C
}

Abdullah Hussain Hamed, Hassan Metwally Bayoumi, Abdulmonem Mohammad Abdulmonem*

Department of Ophthalmology, Faculty of Medicine, Al-Azhar University

*Corresponding author: Abdulmonem Mohammad Abdulmonem; Mobile: (+20) 01060178928;

Email: drabdelmonem2020@ gmail.com

\begin{abstract}
Background: Diabetic retinopathy affects up to $80 \%$ of all patients who have had diabetes for 10 years or more. Despite these intimidating statistics, research indicates that at least $90 \%$ of these new cases could be reduced if there were proper treatment and monitoring of the eyes blindness.

Objective: To evaluate the relation of macular and choroidal thickness to HbAlc in patients with proliferative diabetic retinopathy.

Patients and methods: This study included 40 eyes of 33 patients with a diagnosis of proliferative diabetic retinopathy (PDR). Patients were recruited from Retinal Clinic in Imbaba Eye Hospital and they were asked to participate in this study. This study was designed as an observational, cross-sectional and non-coherent study in the period from 5/2018 to 4/2019.

Results: the results showed that the macular thickness was higher and the choroidal thickness was lower in uncontrolled $\mathrm{HbA} 1 \mathrm{c}$ group than controlled HbA1c group. We assumed that increase level of glycosylated hemoglobin lead to increase macular thickness and decrease choroidal thickness and increase incidence of diabetic macular edema and choroidal atrophy.

Conclusion: Optical coherence tomography is a sensitive and noninvasive diagnostic tool in the evaluation of macular and choroidal thickness. Hypertension is also an important risk factor in the development of diabetic retinopathy, diabetic macular edema and diabetic choroidopathy.

Keywords: Optical Coherence Tomography, Macular and Choroidal Thickness, Proliferative Diabetic Retinopathy, Hemoglobin A1C.
\end{abstract}

\section{INTRODUCTION}

Diabetic retinopathy (DR) is the most common cause of blindness in Europe, affecting $1.9 \%$ of patients with DM. Furthermore, 2.64\% of diabetic patients have visual sight-threatening diabetic retinopathy (STDR) ${ }^{(\mathbf{1})}$.

The prevalence of diabetic retinopathy (DR) remains high at $40 \%$ of diabetic patients. Globally, there are approximately 93 million people with DR, 70 million with proliferative diabetic retinopathy (PDR), 21 million with diabetic macular edema (DME) and 28 million with a sight- threatening retinopathy as proliferative diabetic retinopathy (PDR) (2). Diabetic retinopathy affects up to $80 \%$ of all patients who have had diabetes for 10 years or more ${ }^{(3)}$.

Despite these intimidating statistics, research indicates that at least $90 \%$ of these new cases could be reduced if there were proper and vigilant treatment and monitoring of the eyes ${ }^{(4)}$. The longer a person has diabetes, the higher is his or her chances of developing diabetic retinopathy ${ }^{(5)}$.

Diabetic retinopathy is a progressive disease predominantly affects the integrity of the microscopic vessels found in the retina. DR can be broadly divided into two clinical stages: non proliferative and proliferative diabetic retinopathy $(\mathrm{PDR})^{(\mathbf{6})}$.
Proliferative diabetic retinopathy develops following the occlusion of retinal capillaries leading to retinal ischemia, which promotes the development of neovascularization, a process by which new blood vessels proliferate on the surface of the retina. However, these vessels are fragile and bleed easily. The resulting accumulation of blood in the vitreous cavity from these hemorrhaging vessels seriously impairs vision. This may be permanent due to further complications such as traction retinal detachment leading to registered blindness. It has been estimated that without treatment for PDR, 50\% of all patients will become blind within 5 years following diagnosis ${ }^{(7)}$.

Optical Coherence Tomography (OCT) is a high-resolution, cross-sectional imaging technique that allows detailed assessment of retinal thickness and morphologic evaluation of the neurosensory retinal layers. OCT imaging has rapidly been integrated into diagnosis and management of DME in routine clinical practice and clinical trials ${ }^{(8)}$.

One major advantage of OCT is that it allows measurement of retinal thickness from the tomograms by means of computer imageprocessing techniques, OCT is more sensitive to small changes in retinal thickness than slit-lamp biomicroscopy ${ }^{(9)}$. 
Risk factors that contribute to the progression of DME include increasing level of hyperglycaemia, diabetes duration, severity of diabetic retinopathy at baseline, diastolic blood pressure and the presence of gross proteinuria ${ }^{(\mathbf{1 0})}$.

Periodic glycosylated hemoglobin (HbA1c) measurements can reflect the long-term control of hyperglycemia. Intensive glycemic control had been proved to be effective in decreasing incidence rate of development and progression of diabetic retinopathy in type 1 and type II diabetic mellitus as demonstrates by diabetes control and complication trials ${ }^{(\mathbf{1 1})}$.

\section{AIM OF THE WORK}

To evaluate the relation of macular and choroidal thickness to $\mathrm{HbA1c}$ in patients with proliferative diabetic retinopathy.

\section{PATIENTS AND METHODS}

This study included 40 eyes of 33 patients with a diagnosis of proliferative diabetic retinopathy (PDR). Patients were recruited from Retinal Clinic in Imbaba Eye Hospital they were asked to participate in this study.

This study was designed as an observational, cross-sectional and non-coherent study in the period from 5/2018 to 4/2019.

Ethical approval and written informed consent:

An approval of the study was obtained from Al- Azhar University academic and ethical committee. Every patient signed an informed written consent for acceptance of the operation.

\section{Study Population:}

\section{Patients were divided into 2 groups:}

- Controlled HbA1c group: 20 eyes with proliferative diabetic retinopathy with controlled glycosylated hemoglobin (HbAlc $\leq 7 \%$ ).

- Uncontrolled HbA1c group: 20 eyes with proliferative diabetic retinopathy with uncontrolled glycosylated hemoglobin $(\mathrm{HbAlc}>7 \%)$.

\section{Inclusion criteria:}

Type I and type II proliferative diabetic retinopathy patients (PDR).

\section{Exclusion criteria:}

1- Non proliferative diabetic retinopathy (NPDR).

2- Opaque cornea.

3- Opaque lens.

4- Refraction more than +6 or -6 .

5- Laser, intravitreal injection and intraocular surgery that have been done within 3 months before OCT assessment.

6- Glaucoma.

7- Systemic diseases complicated with significant fluid retention as hypertension, heart failure, renal failure, liver cell failure and chronic obstructive pulmonary disease (COPD).

\section{Study design:}

All subjects participating in the study were asked to sign consent before inclusion. Then they were subjected to:

1. Full Medical history.

2. Blood sample was taken on the day of OCT assessment to measure HbA1c level.

3. Measurements of the IOP, and CMT and SFCT measurements by SD OCT (EDI).

Ocular examination included:

a- Best corrected visual acuity (BCVA) using a Snellen chart.

b- Intraocular pressure by Goldman applanation tonometer.

c- Anterior and posterior segment examination by a slit-lamp biomicroscopy.

d- Dilated fundus examination with both slitlamp biomicroscopy with a 90D lens and indirect ophthalmoscopy.

The proliferative diabetic retinopathy (PDR) was diagnosed according to the simplified Early Treatment Diabetic Retinopathy Study (ETDRS) severity scale (ETDRS Research Group 1991) by the presence of the fibrovascular, proliferation, new vessels elsewhere, and new vessels at $<1$ disc diameter of the disc, preretinal hemorrhage, or vitreous hemorrhage.

\section{SD-OCT scanning protocols}

OCT examination was done to all patients using The Spectralis OCT device (Heidelberg Engineering, Dossenheim, Germany) (software version 5.6.3.0; Heidelberg Engineering). The Spectralis OCT has an acquisition rate of 40,000 A-scans per second. It uses a dual-beam SD-OCT and a confocal scanning laser ophthalmoscope (CSLO) that uses a scanning laser diode with a wavelength of $870 \mathrm{~nm}$ and an infrared reference image simultaneously to provide images of ocular structures. The instrument incorporates a real-time eye tracking system that couples CSLO and SDOCT scanners to adjust for eye motion.

Macular scan was conducted using preset fast scan with macular cube volume of $\left(20^{\circ} \times 20^{\circ}\right)$, with number of scans $25 \mathrm{~A}$-scan and distance between scans $259 \mu \mathrm{m}$ with scan angle $20^{\circ}$. 
The changes in the SFCT were recorded with the EDI-OCT technique, with retinal scans performed along horizontal lines $\left(7\right.$ lines, $30^{\circ} \times$ $10^{\circ}$ ) through the center of the fovea. For the EDIOCT technique, each section was obtained using eye tracking, and 25 scans were averaged to improve the signal-to-noise ratio. The CMT was defined as the distance between the internal limiting membrane (ILM) to the RPE, and the SFCT was defined as the distance between the outer border of the hyperreflective line corresponding to the RPE and the outer border of the choroid beneath the centre of the fovea.

Patients with $\mathrm{HbA} 1 \mathrm{c} \leq 7 \%$ were defined as controlled group and patients with $\mathrm{HbA} 1 \mathrm{c}>$ $7 \%$ were defined as uncontrolled group.

\section{Statistical Analysis:}

\section{Data Management and Analysis:}

The collected patient's data were revised, coded, tabulated and introduced to a PC using statistical package for social sciences (IBM SPSS VERSION 20.0). Data were presented and suitable analysis was done according to the type of data obtained for each parameter.

\section{I- Descriptive Statistics:}

1. Mean and standard deviation (SD).

2. Frequency and percentage of nonnumerical data.

\section{II- Analytical Statistics:}

1. Independent sample t-test was used to assess the statistical significance of the difference of a parametric variable between two independent means of two study groups; while Mann Whiney $\mathbf{U}$ test was used in case of non-parametric variable.

2. Chi square test was used to examine the relationship between two qualitative variables but when the expected count is less than 5 in more than $20 \%$ of the cells; Fisher's Exact Test was used.

3. Pearson Correlation Coefficient ( $r$ ) in parametric variables and Spearman correlation coefficient (Rho) in nonparametric variables was used.

4. P-value: Level of significance: $\mathrm{P}>0.05$ : Non significant (NS) - $p<0.05$ : Significant (S) - $p<0.01$ : Highly significant (HS).

\section{RESULTS}

The demographic characteristics and selected risk factors are presented in table 1 .

Both groups were comparable as regard age, sex, type of DM and BCVA, but controlled HbA1c group had shorter duration of DM, lower HbA1c level, lower center macular thickness (CMT), lower total macular volume and higher subfoveal choroidal thickness than uncontrolled HbA1c group (statistically significant difference between controlled HbA1c group and uncontrolled $\mathrm{HbA1c}$ group $\mathrm{p}<.05)$.

Table (1): Demographic characteristic and selected risk factor for proliferative diabetic retinopathy patients Values are numbers (percentage).

\begin{tabular}{|l|c|c|}
\hline \multicolumn{1}{|c|}{ Characteristic } & Controlled HbA1c group & Uncontrolled HbA1c group \\
\hline Mean (SD) age (years) & $53.66 \pm 8.48$ & $56.87 \pm 6.46$ \\
\hline Number of male patients & $6(30 \%)$ & $4(20 \%)$ \\
\hline Number of female patients & $14(70 \%)$ & $16(80 \%)$ \\
\hline Number of type I DM & $3(15 \%)$ & $2(10 \%)$ \\
\hline Number of type II DM & $17(85 \%)$ & $18(90 \%)$ \\
\hline Mean (SD) duration of DM (years) & $9.44 \pm 6.29$ & $14.31 \pm 6.22$ \\
\hline Mean (SD) HbA1c $(\%)$ & $6.78 \pm 0.22 \%$ & $8.68 \pm 1.07 \%$ \\
\hline Mean $(S D)$ BCVA & $0.18 \pm 0.78$ & $0.19 \pm 0.81$ \\
\hline Mean (SD) CMT $(\mu \mathrm{m})$ & $232.41 \pm 94.96$ & $317.21 \pm 133.66$ \\
\hline Mean $(\mathrm{SD})$ total macular volume & $8.27 \pm 0.89$ & $9.46 \pm 1.53$ \\
\hline Mean (SD) SFCT $(\mu \mathrm{m})$ & $302.45 \pm 28.64$ & $257.76 \pm 39.18$ \\
\hline
\end{tabular}

Table (2): Comparison between controlled HbA1c group and uncontrolled HbA1c group as regard Age.

\begin{tabular}{|c|c|c|c|c|c|}
\hline \multirow{3}{*}{ Variables } & \multicolumn{2}{|c|}{$\begin{array}{c}\text { Controlled } \\
(\text { HbA1c } \leq 7)\end{array}$} & \multicolumn{2}{|c|}{$\begin{array}{c}\text { Uncontrolled } \\
(\text { HbA1c }<\text { 7) }\end{array}$} & \multirow{2}{*}{$\boldsymbol{p}$-value } \\
\cline { 2 - 5 } & Mean & \pm SD & Mean & 士SD & \\
\hline Age & 53.66 & 8.48 & 56.87 & 6.46 & $>0.05$ \\
\hline
\end{tabular}

There was a statistically insignificant difference between controlled HbA1c group and uncontrolled $\mathrm{HbA1c}$ group regarding age. 
Table (3): Comparison between controlled HbA1c group and uncontrolled HbA1c group as regard sex, and type of diabetes mellitus (DM).

\begin{tabular}{|c|c|c|c|c|c|c|}
\hline & \multicolumn{4}{|c|}{ HbA1c_group } & \multirow{3}{*}{$p$-value } \\
\hline & & \multicolumn{2}{|c|}{ Controlled (HbA1c $\leq 7)$} & \multicolumn{2}{|c|}{ Uncontrolled $(\mathrm{HbA1c}>7)$} & \\
\hline & & No. & $\%$ & No. & $\%$ & \\
\hline \multirow{2}{*}{ Sex } & Male & 6 & $30 \%$ & 4 & $20 \%$ & \multirow{2}{*}{$>0.05$} \\
\hline & Female & 14 & $70 \%$ & 16 & $80 \%$ & \\
\hline \multirow{2}{*}{ Type of DM } & Type I & 3 & $15 \%$ & 2 & $10 \%$ & \multirow{2}{*}{$>0.05$} \\
\hline & Type II & 17 & $85 \%$ & 18 & $90 \%$ & \\
\hline
\end{tabular}

There was statistically insignificant difference between controlled HbAlc group and uncontrolled $\mathrm{HbA} 1 \mathrm{c}$ group regarding sex and type of DM. In controlled HbA1c group 65\% patients were normotensive and $35 \%$ patients were hypertensive and in uncontrolled $\mathrm{HbA} 1 \mathrm{c}$ group $25.0 \%$ patients were normotensive and $75.0 \%$ patients were hypertensive, so there was a highly statistically significant difference between controlled $\mathrm{HbA1c}$ group and uncontrolled $\mathrm{HbA1c}$ group regarding $\mathrm{BP}$.

Table (4): Comparison between controlled HbA1c group and uncontrolled HbAlc group as regard best corrected visual acuity (BCVA), intraocular pressure (IOP) and duration of diabetes mellitus (DM).

\begin{tabular}{|l|c|c|c|c|c|}
\hline \multirow{2}{*}{ Variables } & \multicolumn{2}{|c|}{ Controlled (HbA1c $\leq 7)$} & \multicolumn{2}{c|}{ Uncontrolled $($ HbA1c $>$ 7) } & \multirow{2}{*}{-value } \\
\cline { 2 - 5 } & Mean & + SD & Mean & + SD & $>0.05$ \\
\hline BCVA & 0.18 & 0.78 & 0.19 & 0.81 & $>0.02 * *$ \\
IOP & 16.93 & 1.38 & 18.22 & 1.91 & $0.002^{* *}$ \\
\hline Duration & 9.44 & 6.29 & 14.31 & 6.22 & $<0.001^{* *}$ \\
\hline
\end{tabular}

(**) Highly statistically significant at $p<0.01$

There was a highly statistically significant difference between controlled HbA1c group and uncontrolled HbA1c group regarding IOP and duration of DM, but there was statistically insignificant difference between controlled $\mathrm{HbA1c}$ group and uncontrolled $\mathrm{HbA} 1 \mathrm{c}$ group regarding BCVA.

Table (5): Comparison between controlled HbA1c group and uncontrolled HbA1c group as regard average MT, total macular volume, superior inner MT, superior outer MT, temporal inner MT, temporal outer MT, inferior inner MT and nasal inner MT.

\begin{tabular}{|c|c|c|c|c|c|}
\hline \multirow{2}{*}{ Variables } & \multicolumn{2}{|c|}{ Controlled (HbA1C $\leq$ 7) } & \multicolumn{2}{c|}{ Uncontrolled (HbA1C $>$ 7) } & \multirow{2}{*}{$\boldsymbol{p}$-value } \\
\cline { 2 - 5 } & Mean & $\mathbf{+ ~ S D}$ & Mean & + SD & \\
\hline Average MT & 287.52 & 32.02 & 328.24 & 53.45 & $0.006^{* *}$ \\
\hline Total macular volume & 8.27 & 0.89 & 9.46 & 1.53 & $0.005^{* *}$ \\
\hline Superior inner MT & 307.99 & 45.31 & 352.11 & 62.84 & $<0.02^{*}$ \\
\hline Superior outer MT & 284.99 & 38.74 & 321.16 & 49.03 & $0.001^{* *}$ \\
\hline Temporal inner MT & 296.03 & 48.94 & 343.35 & 77.57 & $<0.03^{*}$ \\
\hline Temporal outer MT & 275.62 & 34.78 & 316.13 & 56.05 & $<0.01^{* *}$ \\
\hline Inferior inner MT & 292.60 & 53.28 & 350.39 & 94.57 & $<0.03^{*}$ \\
\hline Nasal inner MT & 303.05 & 63.11 & 351.10 & 86.90 & $>0.05$ \\
\hline
\end{tabular}

(**) Highly statistically significant at $p<.01$

There was a highly statistically significant difference between controlled HbA1c group and uncontrolled HbA1c group as regard Average MT, total macular volume, Superior inner MT, Superior outer MT, Temporal inner MT, Temporal outer MT, Inferior inner MT and Nasal inner MT ( $\mathrm{p}<.01)$. 
Table (6): Comparison between controlled HbA1c group and uncontrolled HbAlc group as regard center macular thickness (CMT), foveal macular thickness (FMT), inferior outer MT and nasal outer MT.

\begin{tabular}{|l|c|c|c|c|c|}
\hline \multirow{2}{*}{\multicolumn{1}{c|}{ Variables }} & \multicolumn{2}{|c|}{ Controlled (HbA1C $\leq$ 7) } & \multicolumn{2}{c|}{ Uncontrolled (HbA1C> 7) } & \multirow{2}{*}{$\boldsymbol{p}$-value } \\
\cline { 2 - 5 } & Mean & + SD & Mean & + SD & \\
\hline Center MT & 232.41 & 94.96 & 317.21 & 133.66 & $0.007^{*}$ \\
\hline Foveal macular thickness & 249.27 & 82.76 & 329.56 & 116.29 & $0.001^{* *}$ \\
\hline inferior outer MT & 282.80 & 33.83 & 317.26 & 82.54 & $0.039^{*}$ \\
\hline nasal outer MT & 298.33 & 44.48 & 331.18 & 67.37 & $0.006^{*}$ \\
\hline
\end{tabular}

(*) Statistically significant at $p<.05,(* *)$ Highly statistically significant at $p<.01$

There is a highly statistically significant difference between controlled HbA1c group and uncontrolled HbA1c group as regard center MT, foveal thickness, inferior outer MT and nasal outer MT $(\mathrm{p}<.05)$.

Table (7): Show comparison between controlled HbA1c group and uncontrolled HbA1c group as regard choroidal thickness (CT).

\begin{tabular}{|l|c|c|c|c|c|}
\hline \multirow{2}{*}{ Distance from fovea } & \multicolumn{2}{|c|}{ Controlled (HbA1c $\leq 7)$} & \multicolumn{2}{|c|}{ Uncontrolled (HbA1c } & \multirow{2}{*}{ > 7) } \\
\cline { 2 - 5 } & Mean & + SD & Mean & + SD & \\
\cline { 2 - 5 } & 275.62 & 34.78 & 241.52 & 41.60 & $<0.01^{* *}$ \\
\hline Temporal $1000 \mu \mathrm{m} \mathrm{CT}$ & 284.36 & 33.18 & 244.40 & 39.89 & $<0.002^{* *}$ \\
\hline Temporal 500 $\mu \mathrm{m} \mathrm{CT}$ & 302.45 & 28.64 & 257.76 & 39.18 & $0.000^{* *}$ \\
\hline Subfoveal CT & 291.02 & 31.63 & 243.89 & 41.55 & $0.000^{* *}$ \\
\hline Nasal $500 \mu \mathrm{m} \mathrm{CT}$ & 286.70 & 32.29 & 240.32 & 46.76 & $0.000^{* *}$ \\
\hline Nasal $1000 \mu \mathrm{m} \mathrm{CT}$ & & &
\end{tabular}

(**) Highly statistically significant at $p<.01$

There was a highly statistically significant difference between controlled HbAlc group and uncontrolled HbA1c group as regard CT measurement subfoveal, at $500 \mu \mathrm{m}$ and $1000 \mu \mathrm{m}$ temporal to fovea and $500 \mu \mathrm{m}$ and $1000 \mu \mathrm{m}$ nasal to fovea $(\mathrm{p}<.01)$.

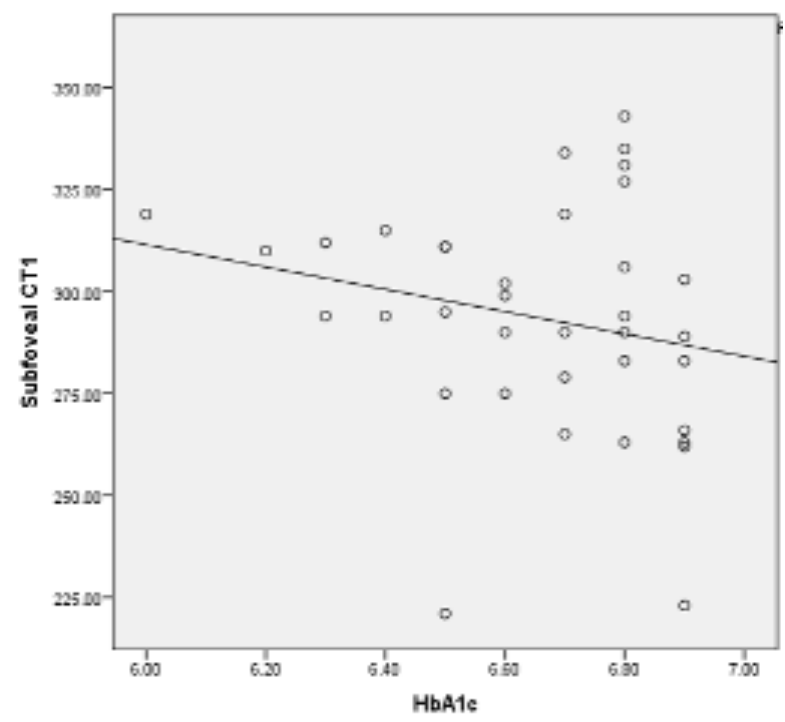

Figure (1): Correlation between glycosylated hemoglobin (HbA1c) and subfoveal choroidal thickness (SFCT) in controlled HbA1c group.

There was weak negative correlation between glycosylated hemoglobin (HbA1c) and subfoveal choroidal thickness (SFCT) in controlled HbAlc group, which was statistically insignificant $(p>.05)$ 


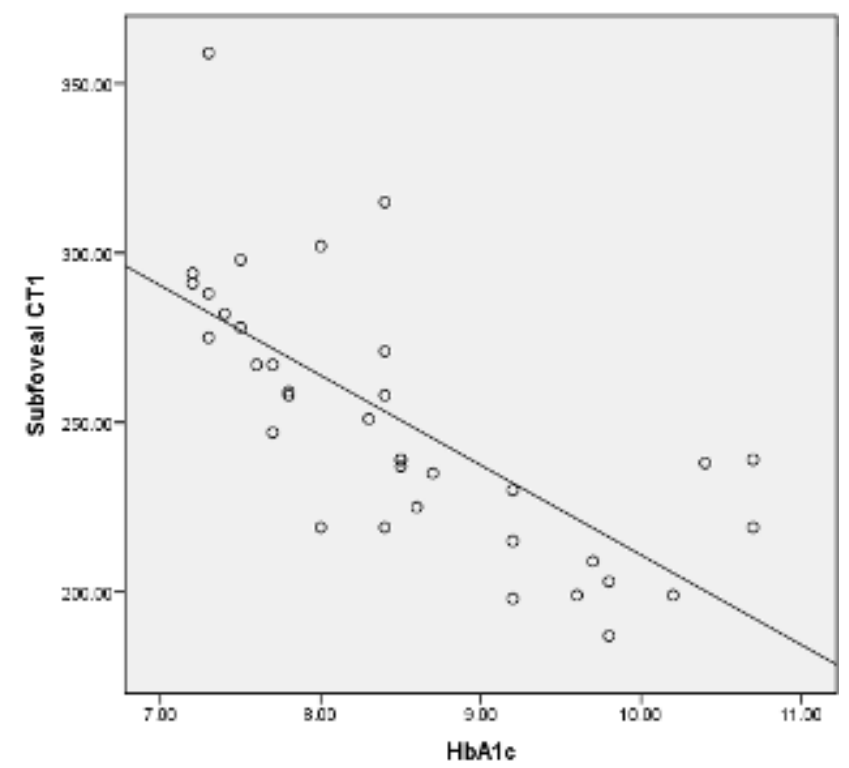

Figure (2): Correlation between glycosylated hemoglobin (HbA1c) and subfoveal choroidal thickness SFCT in uncontrolled $\mathrm{HbA1c}$ group.

There was a strong negative correlation between glycosylated hemoglobin (HbA1c) and subfoveal choroidal thickness (SFCT) in uncontrolled HbA1c group, which was statistically significant $(p>.05)$.

Spearman correlation coefficient test was computed to assess the relationship between foveal macular thickness (FMT) and subfoveal choroidal thickness (SFCT) in controlled HbA1c group (Figure 3) and uncontrolled HbA1c group (Figure 4).

In controlled $\mathrm{HbA} 1 \mathrm{c}$ group, there was a very weak negative correlation between foveal macular thickness (FMT) and subfoveal choroidal thickness (SFCT), which was statistically insignificant ( $r=-.041$, $p=.762$ ).

In uncontrolled $\mathrm{HbA1c}$ group, there was a weak negative correlation between foveal macular thickness (FMT) and subfoveal choroidal thickness (SFCT), which was statistically insignificant $(r=-.249$, $p=.240)$.

The results suggested that increase in FMT correlated with decrease in SFCT.

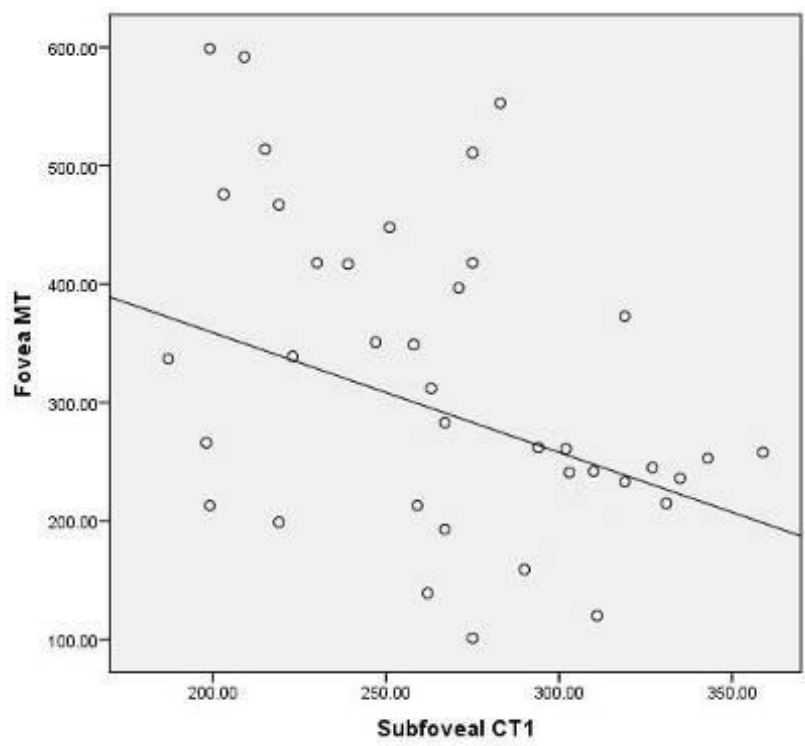

Figure (3): Correlation between subfoveal choroidal thickness (SFCT) and foveal macular thickness (FMT) in controlled $\mathrm{HbA} 1 \mathrm{c}$ group.

There was a very weak negative correlation between subfoveal choroidal thickness (SFCT) and foveal macular thickness (FMT) in controlled HbA1c group, which was statistically insignificant ( $p>.05$ ). 


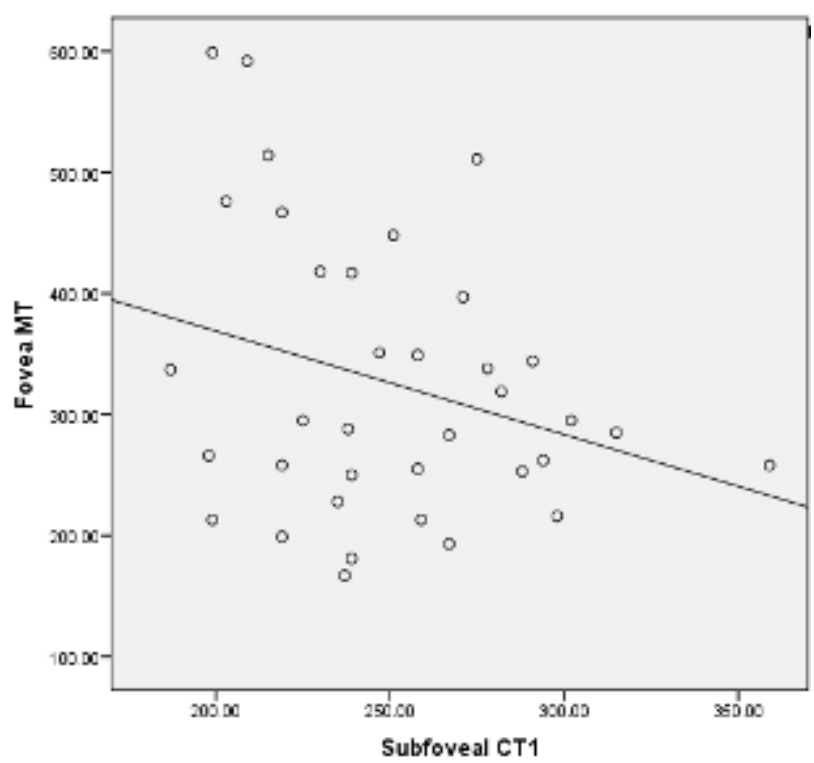

Figure (4): Correlation between subfoveal choroidal thickness (SFCT) and foveal macular thickness (FMT) in uncontrolled $\mathrm{HbA1c}$ group.

There was a weak negative correlation between subfoveal choroidal thickness (SFCT) and foveal macular thickness (FMT) in uncontrolled HbA1c group, which was statistically insignificant ( $p>.05)$.

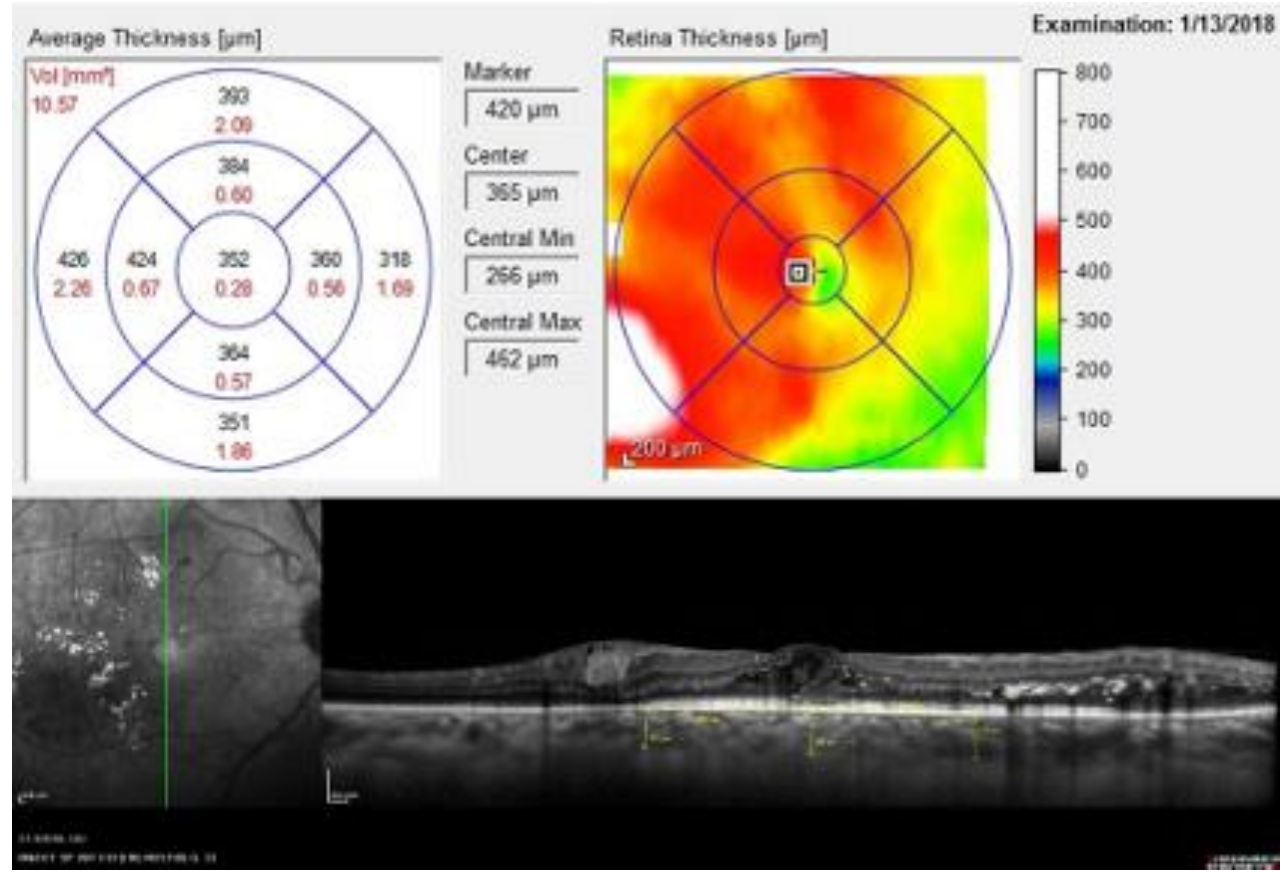

Figure (5): CMT and Choroidal thickness by EDI in Group 1 controlled HA1c 
ejhm.journals.ekb.eg

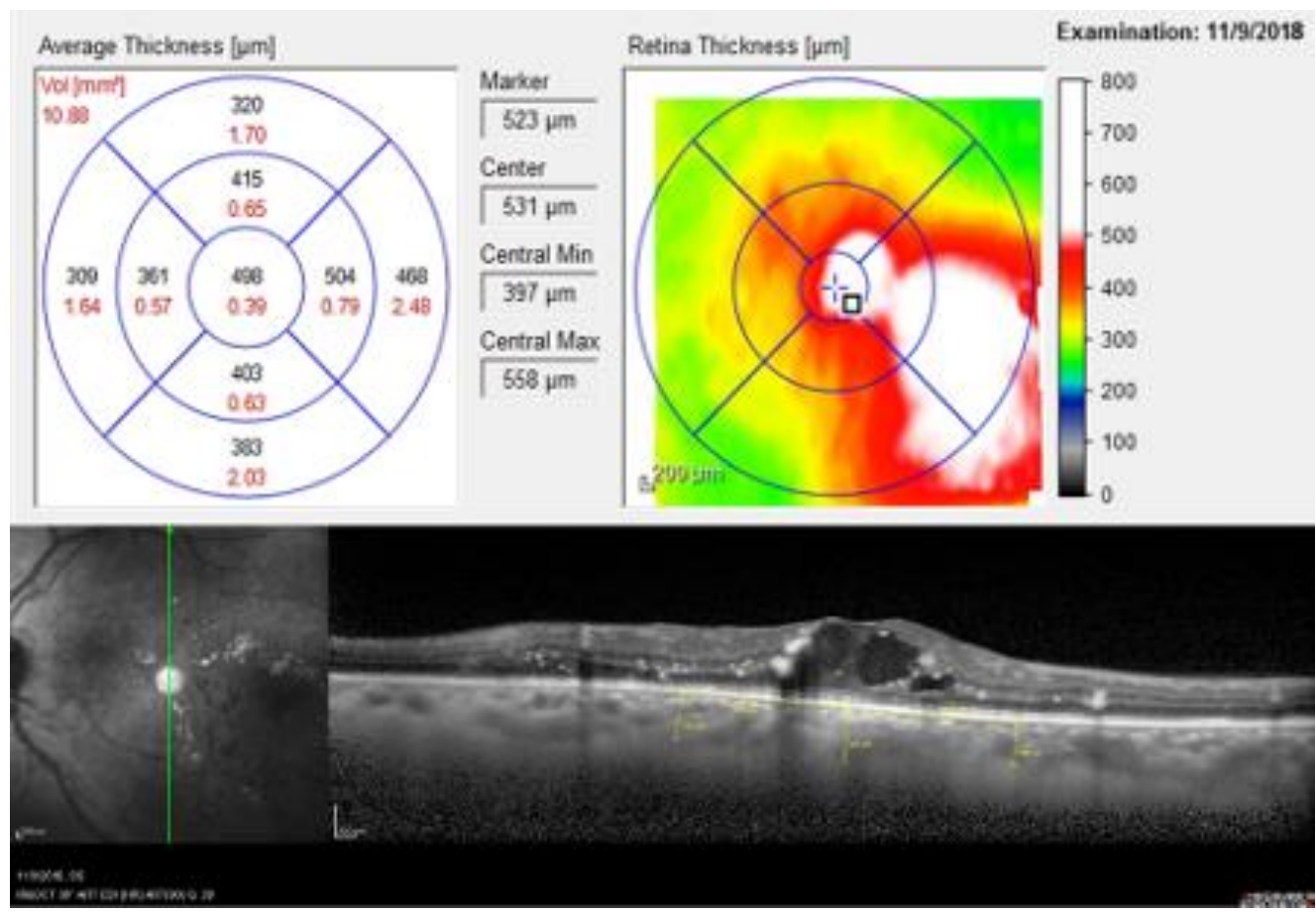

Figure (6): CMT and Choroidal thickness by EDI in Group 1 controlled HA1c

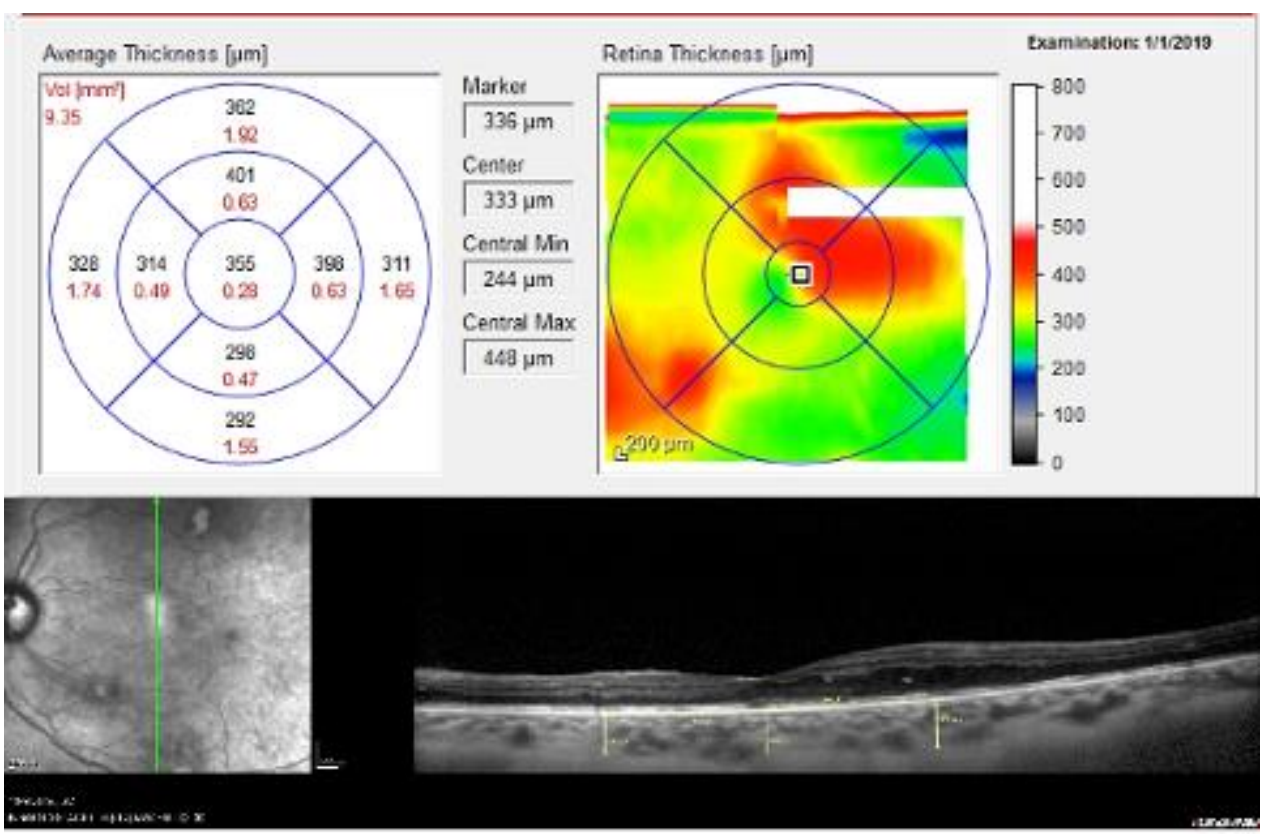

Figure (7): CMT and Choroidal thickness by EDI in Group 1 controlled HA1c 
ejhm.journals.ekb.eg

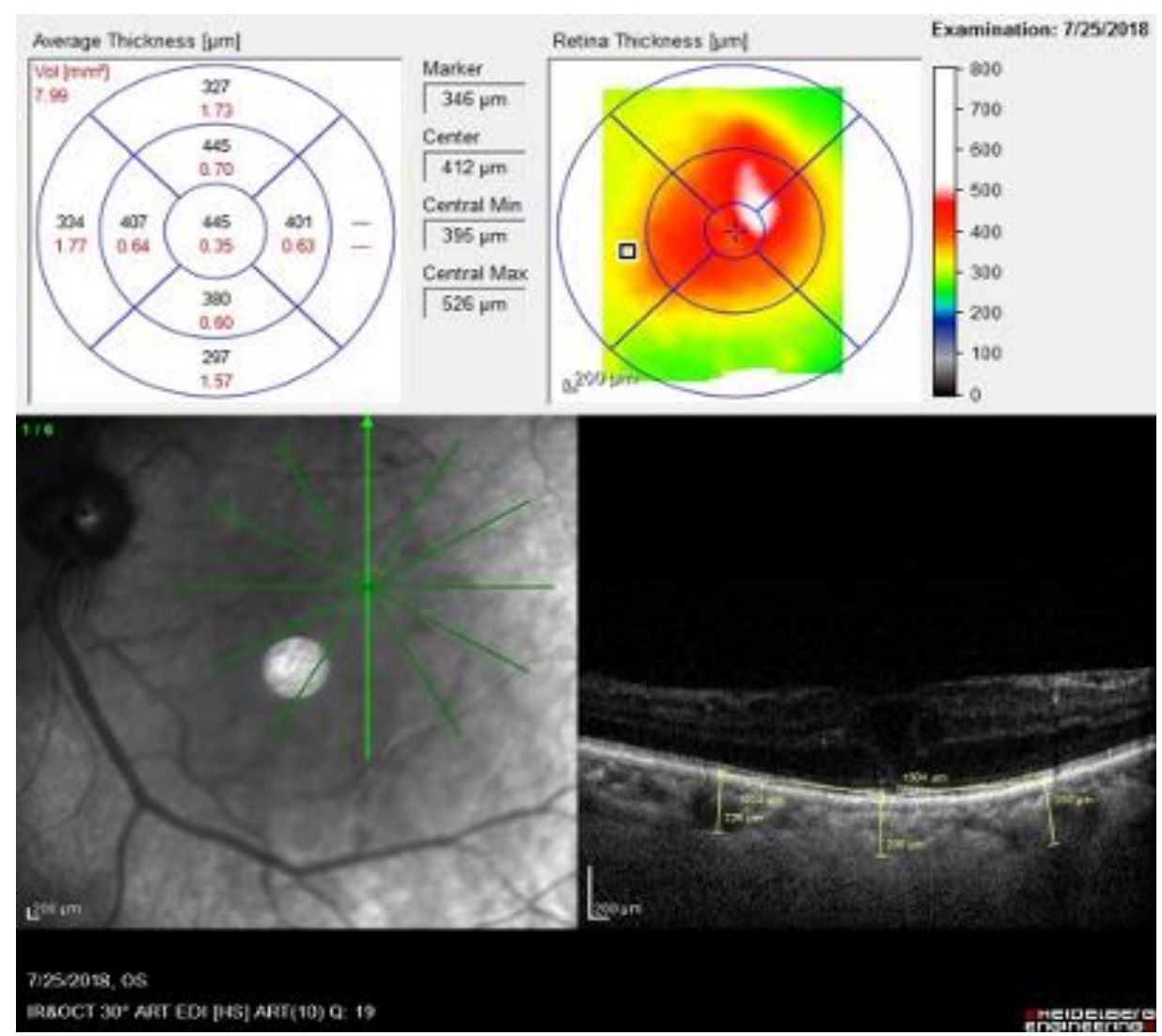

Figure (8): CMT and Choroidal thickness by EDI in Group 2 in uncontrolled HA1c

Table (8): Comparison between time of examination and after 3 months according to all parameters in uncontrolled (HbA1C>7).

\begin{tabular}{|c|c|c|c|c|c|}
\hline \multirow{2}{*}{$\begin{array}{c}\text { Uncontrolled } \\
(\text { HbA1C> 7) }\end{array}$} & \multicolumn{2}{|c|}{ Pre } & \multicolumn{2}{|c|}{ After 3 months } & \multirow{2}{*}{ p-value } \\
\hline & Mean & \pm SD & Mean & \pm SD & \\
\hline BCVA & 0.19 & 0.81 & 0.21 & 0.88 & $>0.05$ \\
\hline IOP & 18.22 & 1.91 & 18.04 & 1.89 & $>0.05$ \\
\hline Average MT & 328.24 & 53.45 & 324.96 & 52.92 & $>0.05$ \\
\hline Total macular volume & 9.46 & 1.53 & 9.37 & 1.51 & $>0.05$ \\
\hline Superior inner MT & 352.11 & 62.84 & 348.59 & 62.21 & $>0.05$ \\
\hline Superior outer MT & 321.16 & 49.03 & 317.95 & 48.54 & $>0.05$ \\
\hline Temporal inner MT & 343.35 & 77.57 & 341.63 & 77.18 & $>0.05$ \\
\hline Temporal outer MT & 316.13 & 56.05 & 314.55 & 55.77 & $>0.05$ \\
\hline Inferior inner MT & 350.39 & 94.57 & 346.89 & 93.62 & $>0.05$ \\
\hline Nasal inner MT & 351.1 & 86.9 & 347.59 & 86.03 & $>0.05$ \\
\hline Center MT & 317.21 & 133.66 & 314.04 & 132.32 & $>0.05$ \\
\hline Foveal macular thickness & 329.56 & 116.29 & 326.26 & 115.13 & $>0.05$ \\
\hline inferior outer MT & 317.26 & 82.54 & 314.09 & 81.71 & $>0.05$ \\
\hline nasal outer MT & 331.18 & 67.37 & 327.87 & 66.70 & $>0.05$ \\
\hline Temporal $1000 \mu \mathrm{m} \mathrm{CT}$ & 241.52 & 41.6 & 244.18 & 42.06 & $>0.05$ \\
\hline Temporal $500 \mu \mathrm{m} \mathrm{CT}$ & 244.4 & 39.89 & 247.09 & 40.33 & $>0.05$ \\
\hline Subfoveal CT & 257.76 & 39.18 & 260.60 & 39.61 & $>0.05$ \\
\hline Nasal $500 \mu \mathrm{m}$ CT & 243.89 & 41.55 & 246.57 & 42.01 & $>0.05$ \\
\hline Nasal $1000 \mu \mathrm{m}$ CT & 240.32 & 46.76 & 242.96 & 47.27 & $>0.05$ \\
\hline
\end{tabular}

This table shows no statistically significant difference between time of examination and after 3 months according to all parameters in uncontrolled (HbA1C $>7)$ after controlling $\mathrm{HbA} 1 \mathrm{C}$. 


\section{DISCUSSION}

In our study the center macular thickness (CMT) was significantly lower in controlled $\mathrm{HbA1c}$ group than uncontrolled $\mathrm{HbAlc}$ group with medium effect size, the total macular volume (TMV) was significantly lower in controlled $\mathrm{HbA1c}$ than uncontrolled $\mathrm{HbA} 1 \mathrm{c}$ with large effect size and the subfoveal choroidal thickness (SFCT) was significantly higher in controlled $\mathrm{HbAlc}$ group than uncontrolled HbA1c group with large effect size.

Diabetic retinopathy (DR) is a complication of diabetes mellitus (DM) and a leading cause of blindness worldwide. DR is generally thought to be caused by abnormalities of retinal microvasculature, but recent studies have shown that the choroid plays an important role in the progress of DR. The choroid is a layer of the eye that provides $95 \%$ of the ocular blood flow; it supplies oxygen and nutrients to the outer retina, including photoreceptors and retinal pigment epithelium, and is the sole provider of blood flow to the avascular fovea. This function of the choroid is affected in DR, in association with choroidal vasculopathy ${ }^{(\mathbf{1 2})}$.

We excluded from the study patients with refraction more than +6 or -6 to exclude the effect of axial length on choroidal thickness (CT), patients had pan retinal photocoagulation (PRP) or intravitreal injection within 3 months before optical coherence tomography (OCT) assessment to exclude their effect on macular thickness (MT) and choroidal thickness (CT) and patients with systemic diseases complicated with significant fluid retention as heart failure, renal failure, liver cell failure, hypertension and chronic obstructive pulmonary disease (COPD) to exclude their effect on CT.

In our study the 2 groups were comparable regarding age and the mean age of the 2 groups was $>50$ years old.

In agreement with our study Lima et al. (13) reported a greater chance of diabetic retinopathy (DR) in individuals aged 50-59 years and $\geq 60$ years and Raman $\boldsymbol{e t}$ al. ${ }^{(\mathbf{1 4})}$ also reported the significance of age as a risk factor for DR.

In contrast to our study Yang et al. ${ }^{(15)}$ reported no association between age and DR.

In our study the 2 groups were comparable regarding sex but in controlled HbA1c $72.2 \%$ patients were female and in uncontrolled $\mathrm{HbA} 1 \mathrm{c}$ group $80.6 \%$ patients were female.

In agreement with our study MaricBilkan ${ }^{(16)}$ reported greater chance of DR among females.
In contrast to our study Raman et al. ${ }^{(14)}$ reported greater chance of DR among males.

In our study the 2 groups were comparable regarding BCVA although we used Snellen chart for visual acuity measurement not $\log$ MAR method.

In our study the mean duration of DM was longer and $\mathrm{HbAlc}$ level was higher in uncontrolled $\mathrm{HbA} 1 \mathrm{c}$ group than controlled $\mathrm{HbA} 1 \mathrm{c}$ group and the macular thickness (MT) was thicker and in uncontrolled $\mathrm{HbA} 1 \mathrm{c}$ group than controlled $\mathrm{HbA1c}$ group.

In agreement with our study RomeroAroca et al. ${ }^{(17)}$ reported that the incidence of macular edema over the 8-year period was associated with higher levels of glycosylated hemoglobin and more severe retinopathy in both younger- and older-onset groups.

Yeoung et al. (18) also reported that $\mathrm{HbA1c}$ level positively correlated with macular thickness in patients with type I and II diabetes of 10 or more year's duration without diabetic macular edema. This study suggests that subclinical macular volume and thickness changes may occur before diabetic macular edema (DMO) becomes clinically evident.

So we could suggest that intensive glycemic control is associated with delaying the development and progression of diabetic retinopathy. HbAlc of 7 or above increased the risk of diabetic macular edema (DME).The duration of diabetes is also a risk factor for development of DME. However, the reported duration of type II DM is usually not reliable due to the non-specific symptoms of DM and difficulty of the patient to recall those symptoms. Some patients were diagnosed with known diabetic complications, indicating that they likely had the disease for years before being diagnosed.

Tuncer et al. ${ }^{(19)}$ reported that CT shows mild decrease with age, so the age may have a minor effect on choroidal thinning.

In our study uncontrolled $\mathrm{HbA1c}$ group with thinner CT also have higher macular thickness (diabetic macular edema) and we included proliferative diabetic retinopathy (PDR) patients who had active neovascularization and we excluded patients who did laser within 3 months before OCT assessment.

In agreement to our study Laíns et al. ${ }^{(20)}$ optical coherence tomography demonstrated a significant reduction of $\mathrm{CT}$ in PDR compared with controls. In the foveal region, the choroid appears to be thinner in DR eyes than in diabetic eyes without retinopathy. Ünsal $\boldsymbol{e t}$ al. (21) also reported that CT decreases as the disease progresses from mild-moderate NPDR to PDR 
and in DME than non DME patients, so we could conclude that the relation between the severity of $\mathrm{HbA1c}$ level and CT in diabetes mellitus, but they included PDR patients who had received PRP treatment and they found the CT was decreased significantly, in contrast to our study we excluded patients who had pan retinal photocoagulation (PRP) within 3 months before the OCT assessment.

Laíns et al. (20) also reported that significant decrease in choroidal thickness was observed in the mild-to-moderate NPDR, severe NPDR and PDR groups and eyes with macular edema showed significantly decreased choroidal thickness compared with the controls. This is in agreement with our study but our study included only PDR patients.

Wang et al. ${ }^{(22)}$ reported that choroidal vascular density and volume are significantly reduced in more advanced stages of diabetic retinopathy. Schocket et al. (23) reported that choroidal volume and choroidal blood flow are significantly reduced in patients with PDR, but no significant correlations were observed between choroidal volume and choroidal blood flow and HbA1c, in contrast to our study in which we found that $\mathrm{CT}$ is significantly higher in controlled $\mathrm{HbA1c}$ group than uncontrolled HbA1c group.

In contrast to our study Kim et al. (24) reported that choroidal thinning in PRP-treated eyes, but eyes with DME had a thicker choroid than those without DME, In addition, they observed progressive thickening of the choroid layer with increasing severity of DR from mild/moderate NPDR to severe NPDR, or from severe NPDR to PDR and some degree of reduced choroidal thickness among eyes with no DR or early NPDR. But they did not enroll hypertension as a risk factor for choroidal thinning because the number of patients with hypertension was too small, statistical analysis could not be conducted. Whereas BP showed no association with CT, but in our study uncontrolled $\mathrm{HbA1c}$ group with thicker MT (DME) and thinner CT 75\% patients were hypertensive patients compared to controlled HbA1c group 36\% patients were hypertensive patients and in our study we included patients with PDR only.

Kim et al. ${ }^{(24)}$ also detect that clinically the stage of PDR was associated with greater risk of systemic vascular complications, such as ischemic heart disease, This close association suggests that increased CT could be interpreted as a marker of compromised systemic vasculature, in contrast to our study we excluded patients with systemic diseases other than DM complicated with significant fluid retention as heart failure, renal failure, liver cell failure and chronic obstructive pulmonary disease (COPD).

We could assumed that the thinner choroid may indicate an overall reduction of choroidal blood flow (although we did not include an objective choroidal blood flow test) in patients with DME and more prominent in PDR patients with uncontrolled $\mathrm{HbAlc}$, as was previously demonstrated with laser Doppler flowmetry and indocyanine green angiography ${ }^{(23)}$.

Therefore, it is likely that the decreased CT may be related to retinal tissue hypoxia, as the choroid is the major source of nutrition for the RPE and outer retinal layers. What is not clear is whether the thinning detected is primary or secondary to overlying retinal ischemia. Laser treatment may make more choroidal thinning also. We exclude patients had PRP treatment within 3 months before OCT assessment and the age may had a minor effect on choroidal thinning since in our study the mean age in controlled $\mathrm{HbA} 1 \mathrm{c}$ group was 52.61 years $\mathrm{SD} \pm 8.31$ and in uncontrolled $\mathrm{HbA} 1 \mathrm{c}$ group was 55.75 years $\mathrm{SD} \pm$ 6.33 .

\section{CONCLUSION}

Intensive glycemic control might affect retinal and choroidal vasculature and decrease ischemia and affect the development and progression of diabetic retinopathy. Glycosylated hemoglobin of 7 or above increase the risk of macular edema and choroidal thinning.

Optical coherence tomography is a sensitive and noninvasive diagnostic tool in the evaluation of macular and choroidal thickness.

Hypertension is also an important risk factor in the development of diabetic retinopathy, diabetic macular edema and diabetic choroidopathy.

\section{RECOMMENDATION}

Periodic optical coherence tomography examination and glycosylated hemoglobin measurement may provide enough information about dynamic state of macular and choroidal thickness.

\section{REFERENCES}

1. Romero-Aroca P, Baget-Bernaldiz M, ParejaRios A et al. (2016): Diabetic Macular Edema Pathophysiology: Vasogenic versus Inflammatory. Journal of Diabetes Research, 2016:2156273.

2. Yau JW, Rogers SL, Kawasaki R et al. (2014): Global prevalence and major risk factors of diabetic retinopathy. Diabetes Care, 35(3): 64-556. 
3. Pradhan T, Pradhan G, Dasmohapatra T et al. (2016): A review of Cuminoside nano medicinePharma cognostic approach to cancer therapeutics. Journal Young Pharmacist, 8(2):61-7.

4. Doshi D, Aniket S, Deep S et al. (2016): Diabetic Retinopathy Detection Using Deep Convolutional Neural Networks. International Conference on Computing, Analytics and Security Trends, 261-66.

5. Sasongko MB, Felicia W, Angela NA et al. (2017): Prevalence of Diabetic Retinopathy and Blindness in Indonesian Adults with Type 2 Diabetes. American Journal of Ophthalmology, 181: 79-87.

6. Fong DS, Lloyd A, Thomas WG et al. (2003): Diabetic Retinopathy. Position Statement. Diabetes Care, 26 (1): 99-102.

7. Hamilton AMP, Ulbig MW and Polkinghorne $P$ (1996): Epidemiology of Diabetic Retinopathy. In: Hamilton AMP, Ulbig MW and Polkinghorne P, eds. Management of Diabetic Retinopathy. London: B.M.J. Publishing Group; Pp. 1-15.

8. Al-latayfeh MM, Sun JK, Aiello LP (2010): Ocular Coherence Tomography and Diabetic Eye Disease. Semin Ophthalmol., 25(5-6): 7-192.

9. Browning DJ, Fraser CM (2005): Regional patterns of sight-threatening diabetic macular edema. Am J Ophthalmol., 140: 117-124.

10.Stitt AW, Lois N, Medina RJ et al. (2013): Advances in our understanding of diabetic retinopathy. Clin Sci (Lond), 125(1):1-17.

11. Moon SW, Kim HY, Kim SW et al. (2011): The change of macular thickness measured by optical coherence tomography in relation to glycemic control in diabetic patients. Graefe's Arch Clin Exp Ophthalmol., 249(6): 839-848.

12.Jeong KD, Jae YP, Bo NK et al. (2019): Assessment of Choroidal Thickness Inside and Outside of Vascular Arcade in Diabetic Retinopathy Eyes Using Spectral-Domain Optical Coherence Tomography. Scientific Reports, 9 (1): 10780-3.

13. Lima VC, Gabriela CC, Maurício CL et al. (2016): Risk Factors for Diabetic Retinopathy: A Case-Control Study. International Journal of Retina and Vitreous, 2 (1): 21-4.
14. Raman R, Vaitheeswaran $K$, Vinita $K$ et al. (2011): Is prevalence of retinopathy related to the age of onset of diabetes?. Ophthlamic Res., 45: 3641 .

15. Yang JY, Kim NK, Lee YJ et al. (2013): Prevalence and factors associated with diabetic retinopathy in a Korean adult population. Diabetes Res Clin Pract., 102(3): 218-24.

16. Maric-Bilkan C (2017): Sex differences in microand macro-vascular complications of diabetes mellitus. Clinical Science, 131(9):833-846.

17. Romero-Aroca P, Riva-Fernandez S, VallsMateu A et al. (2016): Changes Observed in Diabetic Retinopathy: Eight-Year Follow-up of a Spanish Population. British Journal of Ophthalmology, 100 (10): 1366-71.

18. Yeoung L, Sun CC, Ku WC et al. (2010): Associations between chronic glycosylated haemoglogin (HbA1c) level and macular volume in diabetes patients without macular edema. Acta Ophthalmol., 88: 753-758.

19. Tuncer I, Eyyup K, Mehmet O et al. (2015): Choroidal thickness in relation to sex, age, refractive error, and axial length in healthy turkish subjects. International Ophthalmology, 35 (3): 403 10.

20.Laíns I, Katherine ET, Ana RS et al. (2018): Choroidal thickness in diabetic retinopathy assessed with swept-source optical coherence tomography. Retina, 38 (1): 173-82.

21. Ünsal E, Eltutar K, Zirtiloğlu S et al. (2014): Choroidal thickness in patients with diabetic retinopathy. Clin Ophthalmol., 8: 637-642.

22. Wang JC, Inês L, Joana P et al. (2017): Diabetic choroidopathy: choroidal vascular density and volume in diabetic retinopathy with swept-source optical coherence tomography. American Journal of Ophthalmology, 184: 75-83.

23. Schocket LS, Brucker AJ, Niknam RM et al. (2011): Foveolar choroidal hemodynamics in proliferative diabetic retinopathy. Int Ophthalmol., 25: 89-94.

24. Kim JT, Lee DH, Joe SG et al. (2012): Changes in choroidal thickness in relation to the severity of retinopathy and macular edema in type 2 diabetic patients. Invest Ophthalmol., 54: 3378-3384. 